stages of simian immunodeficiency virus infection compared to peripheral lymphocytes.J Virol. 1998;72(8):6646-6656.

3. Mehandru S, et al. Primary HIV-1 infection is associated with preferential depletion of $\mathrm{CD}^{+} \mathrm{T}$ lymphocytes from effector sites in the gastrointestinal tract. J Exp Med. 2004; 200(6):761-770.

4. Giorgi JV, et al. Shorter survival in advanced human immunodeficiency virus type 1 infection is more closely associated with $\mathrm{T}$ lymphocyte activation than with plasma virus burden or virus chemokine coreceptor usage. J Infect Dis. 1999;179(4):859-870.

5. Sodora DL, Silvestri G. Immune activation and AIDS pathogenesis. AIDS. 2008;22(4):439-446.

6. Gordon SN, et al. 2007. Severe depletion of mucosal $\mathrm{CD}^{+} \mathrm{T}$ cells in AIDS-free simian immunodeficiency virus-infected sooty mangabeys. J Immunol. 2007;179(5):3026-3034.

7. Silvestri G, et al. Nonpathogenic SIV infection of sooty mangabeys is characterized by limited bystander immunopathology despite chronic highlevel viremia. Immunity. 2003;18(3):441-452.

8. Sandler NG, Douek DC. Microbial translocation in HIV infection: causes, consequences and treatment opportunities. Nat Rev Microbiol. 2012;10(9):655-666.

9. Jiang W, et al. Plasma levels of bacterial DNA correlate with immune activation and the magnitude of immune restoration in persons with antiretroviral-treated HIV infection. J Infect Dis. 2009;199(8):1177-1185

10. Nowroozalizadeh S, et al. Microbial translocation correlates with the severity of both HIV-1 and HIV-2 infections. J Infect Dis. 2010;201(8):1150-1154.

11. Epple HJ, et al. Acute HIV infection induces mucosal infiltration with $\mathrm{CD}^{+}$and $\mathrm{CD}^{+}{ }^{+} \mathrm{T}$ cells, epithelial apoptosis, and a mucosal barrier defect. Gastroenterology. 2010;139(4):1289-1300.

12. Korn T, Bettelli E, Oukka M, Kuchroo VK. IL-17 and Th17 cells. Annu Rev Immunol. 2009;27:485-517.

13. Brenchley JM, et al. Differential Th17 CD4 T-cell depletion in pathogenic and nonpathogenic lentiviral infections. Blood. 2008;112(7):2826-2835.

14. Pandrea I, et al. Cutting edge: experimentally induced immune activation in natural hosts of simian immunodeficiency virus induces significant increases in viral replication and $\mathrm{CD}^{+} \mathrm{T}$ cell depletion. J Immunol. 2008;181(10):6687-6691.

15. Kristoff J, et al. Early microbial translocation block- ade reduces SIV-mediated inflammation and viral replication. J Clin Invest. 2014;124(6):2802-2806.

16. Sun PP, Perianayagam MC, Jaber BL. Endotoxin-binding affinity of sevelamer: a potential novel anti-inflammatory mechanism. Kidney Int Suppl. 2009;76(114):S20-S25.

17. Estes J, et al. Collagen deposition limits immune reconstitution in the gut. $J$ Infect Dis. 2008; 198(4):456-464.

18. Mehandru S, et al. Lack of mucosal immune reconstitution during prolonged treatment of acute and early HIV-1 infection. PLoS Med. 2006;3(12):e484.

19. Brenchley JM, et al. Microbial translocation is a cause of systemic immune activation in chronic HIV infection. Nat Med. 2006;12(12):1365-1371.

20. Baroncelli S, et al. Microbial translocation is associated with residual viral replication in HAARTtreated $\mathrm{HIV}^{+}$subjects with $<50$ copies/ml HIV-1 RNA.J Clin Virol. 2009;46(4):367-370.

21. Marchetti G, et al. Microbial translocation is associated with sustained failure in $\mathrm{CD}^{+} \mathrm{T}$-cell reconstitution in HIV-infected patients on longterm highly active antiretroviral therapy. AIDS. 2008;22(15):2035-2038.

\title{
Targeting secondary immune responses to cetuximab: CD137 and the outside story
}

\author{
Julie E. Bauman ${ }^{1}$ and Jennifer R. Grandis²
}

${ }^{1}$ Department of Internal Medicine (Hematology/Oncology) and 2Department of Otolaryngology, University of Pittsburgh, Pittsburgh, Pennsylvania, USA.

\begin{abstract}
Cetuximab is a murine-human chimeric IgG1 $\mathrm{mAb}$ directed against the EGFR that is approved for use in patients with colorectal and head and neck carcinomas. While some patients benefit greatly from cetuximab, many do not; therefore, strategies to increase the efficacy of this drug are of great clinical interest. In this issue of the JCI, Kohrt and colleagues report a strategy for enhancing the secondary immune response to cetuximab that involves sequential targeting with an agonist $\mathrm{mAb}$ against CD137 expressed on NK and T cells.
\end{abstract}

\section{The inside story on cetuximab}

EGFR, also known as HER1, is a member of the erythroblastic leukemia viral oncogene homolog (ERBB) family of transmembrane receptor tyrosine kinases (RTK). Deregulation of EGFR signaling initiates aberrant growth, proliferation, and angiogenesis. The clinical development of mAbs targeting the extracellular ligand-binding domain and small molecules targeting the intracellular ATPase domain of EGFR was originally compelled by the association of EGFR overexpression with poor prognosis in multiple epithelial malignancies. Despite overexpression of EGFR in the vast major-

Conflict of interest: Jennifer Grandis receives laboratory research funding from Novartis.

Citation for this article: J Clin Invest. 2014; 124(6):2371-2375. doi:10.1172/JCI76264. ity of human aerodigestive cancers, cetuximab has only gained FDA approval for use in patients with head and neck squamous cell carcinoma (HNSCC) and WT KRAS colorectal cancer (CRC), with monotherapeutic activity observed in just $10 \%$ to $20 \%$ of patients. Clinical selection for cetuximab is confounded by the absence of a reliable biomarker to predict patient response. Mutations that result in constitutively activated EGFR are associated with patient responsiveness to small-molecule EGFR inhibitors in lung cancer; however, these mutations are rarely identified in HNSCC or CRC. Moreover, neither EGFR overexpression nor EGFR amplification predicts clinical benefit from cetuximab $(1,2)$. The discordance between EGFR target expression and the efficacy of target blockade by cetuximab has broadened investigation into the mechanisms of action and development of therapeutic resistance.

Initial strategies to enhance cetuximab activity have focused on the intracellular signaling hypothesis (Figure 1A), which suggests that de novo or compensatory activation of parallel RTKs (alternate HER family members, cMet, IGF1R, FGFR, VEGFR), downstream EGFR-signaling nodes (RAS, PI3K, STAT3, SRC), or cell cycle promoters (aurora kinase, CDK4/6) circumvents EGFR blockade in HNSCC preclinical models; therefore, coinhibition of these resistance nodes should enhance the activity of cetuximab (3). Cetuximab resistance has also been attributed to heterodimerization of EGFR with other HER proteins that potentially prevent recognition of EGFR by cetuximab as well as acquisition of gain-of-function PIK3CA mutations that activate signaling downstream of EGFR. In CRC patients, activating KRAS and BRAF mutations confer clinical cetuximab resistance. Progressive insight into the complexity and plasticity of the EGFR signaling network has propelled cetuximab-combination trials to evaluate the efficacy of cotargeting these purported resistance nodes (Table 1). 
A

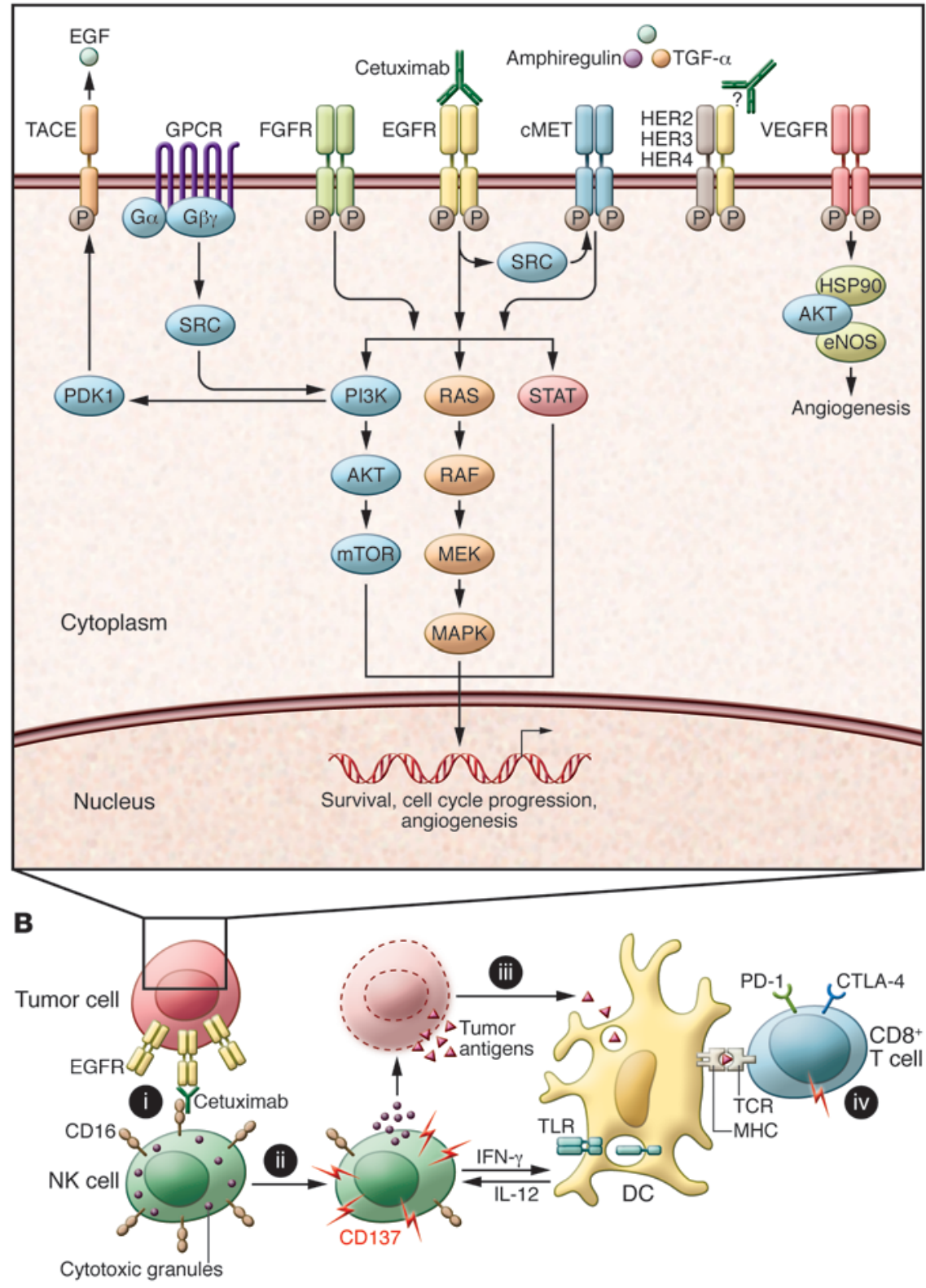

\section{Figure 1}

Intracellular and extracellular approaches to increasing cetuximab efficacy. (A) The inside story. Cetuximab binds to and inhibits EGFR, preventing binding of EGFR ligands and EGFR-dependent activation of cancer-promoting pathways. Blockade of EGFR signaling can be circumvented by crossactivation of accessory RTKs, such as FGFR, cMET, and VEGFR, GPCR signaling, or EGFR-independent activation of any signaling node downstream of EGFR. Cetuximab is being investigated in combination with agents to block other cancer-associated signaling pathways in order to boost efficacy. (B) The outside story. (i) The exposed Fc region of cetuximab bound to EGFR on tumor cells interacts with CD16 on the NK cell surface, promoting NK cell activation. (ii) Once activated, NK cells upregulate CD137 and produce IFN- $\gamma$, which promotes DC maturation. Additionally, NK activation results in cytotoxic degranulation, resulting in tumor cell lysis and the release of TAs. (iii) TAs are taken up by DCs, which present the antigens to CD8 ${ }^{+} \mathrm{T}$ cells (iv). Cetuximab induces both innate and adaptive immune responses. Strategies aimed to amplify the immunologic efficacy of cetuximab enhance NK cell activation, antigen processing and presentation by $\mathrm{DCs}$, or T cell activation.

\section{An outside perspective on blocking EGFR with cetuximab}

Two observations in HNSCC stimulated the search for extracellular immune mechanisms of cetuximab (Figure 1B). First, despite their demonstrated abrogation of EGFR signaling, nonimmunogenic small-molecule inhibitors have not shown clinical efficacy in randomized trials. Second, although both EGFR phosphorylation and tumor proliferation are curtailed in response to cetuximab in vitro, apoptosis or lysis of tumor cells requires coculture with lymphocytes (4). Immune modeling suggests that cetuximab induces sequential innate and adaptive immune responses (5).
These models indicate that EGFR serves as a tumor antigen (TA) that is bound by the variable fragment $(\mathrm{Fab})$ of cetuximab, leaving the exposed IgG1 constant fragment $(\mathrm{Fc})$ on cetuximab-coated cells able to bind Fc $\gamma$ R IIIa (CD16) on NK cells. Fc-CD16 binding then triggers antibody-dependent cell-mediated cytotoxicity (ADCC). In vitro, effective cetuximab-mediated ADCC depends upon IgG1 isotype, Fc fragment glycosylation, and CD16 polymorphisms, which influence the strength of the bond between Fc and CD16 $(4,6)$. Crosslinking of Fc with CD16 activates NK cells and upregulates expression of the costimulatory receptor CD137, production of IFN- $\gamma$, and cytotoxicity. In turn, activated NK cells induce IFN- $\gamma$-dependent DC maturation, enhancing antigen presentation and crosspriming of EGFR-specific $\mathrm{CD}^{+}{ }^{+}$cytotoxic T lymphocytes (7). In theory, strategies to amplify cetuximab-induced NK cell activation would stimulate both innate and adaptive immunity, the latter required for long-lasting immune protection.

\section{A sequential approach to enhancing cetuximab efficacy}

Kohrt and colleagues present evidence that sequential administration of cetuximab followed by an agonistic anti-CD137 $\mathrm{mAb}$ potentiates NK cell degranulation 
Table 1

Cetuximab-combination trials

\begin{tabular}{|c|c|c|}
\hline Target & Drugs & NCT identifier (anatomic site) \\
\hline \multirow[t]{3}{*}{ PI3-kinase } & PX-866 & NCT01252628 (HNSCC) \\
\hline & BYL719 & $\begin{array}{c}\text { NCT01602315 (HNSCC), NCT01719380 } \\
\text { (BRAFmut CRC) }\end{array}$ \\
\hline & BKM120 & NCT01816984 (HNSCC) \\
\hline \multirow[t]{3}{*}{ cMet } & Tivantinib & NCT01602315 (HNSCC) \\
\hline & & NCT01892527 (HNSCC) \\
\hline & & NCT00957853 (CRC) \\
\hline \multirow[t]{3}{*}{ VEGFR } & Bevacizumab & NCT00409565 (HNSCC) \\
\hline & Pazopanib & NCT01716416 (HNSCC) \\
\hline & BAY 43-9006 & NCT00326495 (CRC) \\
\hline \multirow[t]{2}{*}{ HER2 } & Neratinib & NCT01960023 (CRC) \\
\hline & Afatinib & NCT01540682 (solid tumors) \\
\hline \multirow[t]{3}{*}{ HER3 } & R05479599 & NCT01482377 (HER3+ solid tumors) \\
\hline & REGN1400 & NCT01727869 (CRC, HNSCC) \\
\hline & ABT-700 & $\begin{array}{c}\text { NCT01472016 (FOLFIRI-cetuximab } \\
\text { eligible solid tumors) }\end{array}$ \\
\hline HER4 & Afatinib & NCT02020577 (solid tumors) \\
\hline \multirow[t]{2}{*}{ IGF-1R } & Cixutumumab & NCT00957853 (HNSCC) \\
\hline & OSI 906 & NCT01427205 (HNSCC) \\
\hline RAF & BAY 43-9006 & NCT00326495 (CRC) \\
\hline \multirow[t]{2}{*}{ BRAF V600E } & LGX818 & NCT01719380 (BRAFmut CRC) \\
\hline & Vemurafenib & NCT01524978 (BRAFmut CRC) \\
\hline \multirow[t]{2}{*}{ SRC } & Dasatinib & NCT01488318 (HNSCC) \\
\hline & & NCT00882583 (HNSCC) \\
\hline \multirow[t]{3}{*}{ mTOR } & Temsirolimus & $\begin{array}{l}\text { NCT00593060 (CRC), } \\
\text { NCT01256385 (HNSCC) }\end{array}$ \\
\hline & Sirolimus & NCT00940381 (solid tumors) \\
\hline & Everolimus & NCT01133678 (HNSCC) \\
\hline HSP90 & AUY922 & NCT01294826 (CRC) \\
\hline CDK 4/6 & PD 0332991 & NCT02101034 (HNSCC) \\
\hline Aurora kinase & MLN8237 & NCT01540682 (HNSCC) \\
\hline
\end{tabular}

Augmenting ADCC, DC activation, and T cell crosspriming

$\begin{array}{lcc}\text { Target } & \text { Drugs } & \text { NCT identifier (anatomic site) } \\ \text { CD137 agonist } & \text { BMS-663513 } & \text { Pending (HNSCC, CRC) } \\ & \text { PF-05082566 } & \\ \text { TLR agonists } & \text { VTX-2337 (TLR8) } & \text { NCT01334177 (HNSCC) } \\ & & \text { NCT01836029 (HNSCC) } \\ & \text { EMD 1201081 (TLR9) } & \text { NCT01040832 (HNSCC) } \\ \text { Cytokine milieu } & \text { Recombinant IL-12 } & \text { NCT01468896 (HNSCC) } \\ \text { FC-CD16 binding } & \text { CetuGEX } & \text { NCT02052960 (HNSCC monotherapy) }\end{array}$

Overcoming T cell anergy

$\begin{array}{lcc}\text { Target } & \text { Drugs } & \text { NCT identifier (anatomic site) } \\ \text { CTLA-4 } & \text { Ipilimumab } & \text { NCT01860430 (HNSCC) } \\ \text { PD-1/PD-L1 } & \text { BMS-936558 } & \\ & \text { BMS-936559 } & \\ \text { Treg depletion } & \text { Cyclosporine } & \text { NCT01581970 (HNSCC) }\end{array}$

and cytotoxicity against EGFR-expressing HNSCC, mutant KRAS CRC, and WT KRAS CRC cell lines in vitro and as xenografts in murine models (8). A major limitation of many murine xenograft mod- els $(9,10)$ is the use of immunosuppressed animals, which limits analysis to the innate immune response; however, Kohrt et al. evaluated the effectiveness of cetuximab/ anti-CD137 combination therapy against syngeneic xenografts in immune-competent BALB/c mice, using an engineered murine cell line (TUBO) transfected with human EGFR (TUBO-EGFR) (6). While NK cells were necessary for initiation of the therapeutic effect of cetuximab against TUBO-EGFR, depletion of $\mathrm{CD}^{+} \mathrm{T}$ cells also abrogated efficacy. Importantly, $\mathrm{CD}^{+}$ $\mathrm{T}$ cells were required to mediate the memory response and epitope spreading that resulted in rejection of TUBO and TUBOEGFR xenografts both in mice previously cured with combination therapy and in untreated mice that received adoptively transferred $\mathrm{CD}^{+}$splenocytes from cured animals. Kohrt and colleagues also determined that CD137 is upregulated on intratumoral and peripheral NK cells from patients with HNSCC treated with cetuximab, highlighting the translational potential of this therapeutic approach (8).

CD137 is a member of the costimulatory family of TNF receptors that is induced upon activation of $\mathrm{NK}$ and $\mathrm{T}$ cells. Its ligand, CD137L, is expressed on macrophages, activated B cells, and DCs. Endogenous ligation enhances ADCC by NK cells, CD28-dependent activation of the $\mathrm{T}$ cell receptor, and expansion/memory differentiation of $\mathrm{CD}^{+} \mathrm{T}$ cells while inhibiting the immunosuppressive function of $\mathrm{CD}^{+}$Tregs (11). Additionally, CD137 can be stimulated exogenously by engineered mAbs. Enthusiasm for CD137-based immunotherapy emerged after preclinical studies demonstrated that administration of an anti-CD137 mAb eradicated murine sarcomas and mastocytomas in immune-competent animals and was associated with expansion of $\mathrm{CD}^{+}$ TA-specific T cells (12). In tumors that were not responsive to $\mathrm{CD} 137$, a combination of TA-derived peptide vaccine plus anti-CD137 mAb broke T cell naiveté, promoting tumor regression. These results underscored the possibility that poorly immunogenic tumors, similar to human epithelial malignancies, would require combinatorial approaches in which $\mathrm{T}$ cell activation is followed by CD137 agonism. The approach used by Kohrt and colleagues combines cetuximab, which primes NK cell activation by Fc-CD16 crosslinking, and anti-CD137 mAb, which augments both ADCC and the T cell response to generate immunologic memory (8). Results from other preclinical studies targeting HER2-expressing breast cancer with trastuzumab/anti-CD137 and CD20+ lymphoma with rituximab/anti-CD137 suggest that 
sequential therapy with an IgG1, TA-targeting $\mathrm{mAb}$ and an anti-CD137 mAb may be broadly applicable $(9,10)$.

Kohrt and colleagues hypothesized that combined TA-targeting and anti-CD137 $\mathrm{mAb}$ would be less toxic than alternative NK cell-stimulating strategies, such as exogenous cytokine administration, due to restriction of NK activation to the TA-expressing tumor microenvironment (8); however, clinical experience tempers this conclusion. Two humanized, agonist anti-CD137 mAbs (BMS-663513 and PF-05082566) have entered clinical development. While phase I trials for BMS663513 suggested clinical activity, $11 \%$ of patients exhibited serious, reversible liver inflammation, an on-target toxicity likely due to polyclonal $\mathrm{T}$ cell infiltration (13, 14). Furthermore, development of BMS663513 was temporarily suspended during a follow-up phase II trial, when two hepatic fatalities occurred in cohorts receiving a dose greater than $1 \mathrm{mg} / \mathrm{kg}$. Because the biologic activity of BMS-663513 was evident at doses logarithmically lower that $1 \mathrm{mg} /$ $\mathrm{kg}$, phase I trials to evaluate doses in the 0.03 to $0.3 \mathrm{mg} / \mathrm{kg}$ range are now recruiting. Safety data will be necessary before planned combination trials with TA-targeting mAbs proceed.

\section{Limitations and future directions}

While a combinatorial therapeutic approach remains promising, certain translational limitations must be acknowledged. First, the work by Kohrt and colleagues appears agnostic to the intracellular and genomic context of EGFR inhibition (8). Cell lines were selected on the basis of EGFR expression, including SCC4 and SCC6 (HNSCC), PC1 (pancreatic cancer), and T84 and HCT116 G13D (WT KRAS and mutant KRAS CRC), as ADCC activity correlates with TA concentration but not KRAS mutational status (15). EGFR expression also correlates with response to EGFR inhibition in vitro, and HNSCC xenografts in athymic nude mice are uniformly sensitive to cetuximab (16); therefore, the rationale for use of these cell lines is somewhat unclear, and the modest effect of cetuximab monotherapy in the SCC6 xenograft model is surprising. Better characterization of altered intracellular signaling would enhance understanding of the overall therapeutic response to cetuximab, as EGFR-signaling blockade has multiple effects on the immune response, including inhibition of TLR3, decreased EGFR- dependent STAT3 signaling, and enhanced function of EGFR 875 -889-reactive $\mathrm{CD}^{+}{ }^{+} \mathrm{Th}$ cells, which promote $\mathrm{CD}^{+} \mathrm{T}$ cell responses against common ERBB and cMET epitopes $(17,18)$. Second, while Kohrt et al. describe several mutations in genes other than KRAS within the chosen cell lines, this information does not clearly inform the design or interpretation of experiments (8). Unlike in CRC, no link between a particular genetic mutation and clinical cetuximab resistance in HNSCC has been identified. Furthermore, the paucity of genetically engineered HNSCC mouse models limits identification of predictive biomarkers. Given their resemblance to human tumors, patient-derived tumor grafts are promising models for evaluating cetuximab resistance (16). Third, evaluation of the response of tumorigenic human cells in mice is only possible in the context of a compromised host immune system, where only innate immunity persists. Kohrt and colleagues addressed this limitation by using the TUBO-EGFR murine tumor cell line in immune-competent animals (8). Of note, the TUBO progenitor is HER2 dependent, and TUBO-EGFR cells are more responsive to trastuzumab than cetuximab (6). Given the known heterodimerization of EGFR and HER2 and efficacy of dual EGFR/HER2 inhibition in HNSCC models, HER2 expression in TUBO-EGFR cells may influence the signaling response. Additionally, the therapeutic results from an immune-competent mouse model may not translate to human patients. Despite a striking conservation of transcriptional programs during murine and human immune system differentiation, several hundred immune genes diverge and their regulatory mechanisms are less well conserved (19). The ongoing technologic advances in engrafting Il2rg mutated, immunodeficient mice with functional human immune systems will likely provide greater translational confidence (20).

Despite acknowledged limitations, this study by Kohrt and colleagues introduces a new therapeutic paradigm. Much like the EGFR signaling network, the immune cascade following TA-targeting $\mathrm{mAb}$ treatment contains key nodes that can be targeted to amplify the antitumor immune response. Multiple approaches have entered human clinical trials to enhance cetuximab-dependent responses, including stimulation of ADCC with Fc fragment glycoengineering, CD137 agonists, or IL-12, activation of DCs with TLR agonists, and activation of $\mathrm{CD}^{+} \mathrm{T}$ cells with CD137 agonists, suppression of Tregs, or blockade of the coinhibitory receptors CTLA- 4 and PD-1 (Table 1). Ultimately, evaluation of immunotherapy strategies against genetically defined human cancers in humanized mice will permit synthesis of the inside and outside stories and accelerate bedside translation.

\section{Acknowledgments}

This work was supported by the University of Pittsburgh Cancer Institute (UPCI) Head and Neck Cancer SPORE (P50CA097190), the UPCI Cancer Center support grant (P30CA47904), and the American Cancer Society Distinguished Professorship (to J.R. Grandis).

Address correspondence to: Julie E. Bauman, University of Pittsburgh Cancer Institute, 5150 Centre Ave., 5th Floor, Pittsburgh, Pennsylvania 15232, USA. Phone: 412.623.7833; Fax: 412.648.6579; E-mail: baumanje@upmc.edu.

1. Licitra L, et al. Predictive value of epidermal growth factor receptor expression for first-line chemotherapy plus cetuximab in patients with head and neck and colorectal cancer: Analysis of data from the EXTREME and CRYSTAL studies. Eur J Cancer. 2013;49(6):1161-1168.

2. Licitra L, et al. Evaluation of EGFR gene copy number as a predictive biomarker for the efficacy of cetuximab in combination with chemotherapy in the first-line treatment of recurrent and/or metastatic squamous cell carcinoma of the head and neck: EXTREME study. Ann Oncol. 2011;22(5):1078-1087.

3. Burtness B, Bauman JE, Galloway T. Novel targets in HPV-negative head and neck cancer: overcoming resistance to EGFR inhibition. Lancet Oncol. 2013;14(8):e302-e309.

4. Lopez-Albaitero A, et al. Role of antigen-processing machinery in the in vitro resistance of squamous cell carcinoma of the head and neck cells to recognition by CTL. J Immunol. 2006; 176(6):3402-3409.

5. Yang X, Zhang X, Mortenson ED, Radkevich-Brown O, Wang Y, Fu YX. Cetuximab-mediated tumor regression depends on innate and adaptive immune responses. Mol Ther. 2013;21(1):91-100.

6. Patel D, et al. IgG isotype, glycosylation, and EGFR expression determine the induction of antibody-dependent cellular cytotoxicity in vitro by cetuximab. Hum Antibodies. 2010;19(4):89-99.

7. Leemans CR, Braakhuis BJ, Brakenhoff RH. The molecular biology of head and neck cancer. Nat Rev Cancer. 2011;11(1):9-22.

8. Kohrt HE, et al. Targeting CD137 enhances the efficacy of cetuximab. J Clin Invest. 2014; 124(6):2668-2682.

9. Kohrt HE, et al. CD137 stimulation enhances the antilymphoma activity of anti-CD20 antibodies. Blood. 2011;117(8):2423-2432.

10. Kohrt HE, et al. Stimulation of natural killer cells with a CD137-specific antibody enhances trastuzumab efficacy in xenotransplant models of breast cancer. J Clin Invest. 2012;122(3):1066-1075.

11. Melero I, Hirschhorn-Cymerman D, Morales- 
Kastresana A, Sanmamed MF, Wolchok JD. Agonist antibodies to TNFR molecules that costimulate T and NK cells. Clin Cancer Res. 2013;19(5):1044-1053.

12. Melero I, et al. Monoclonal antibodies against the 4-1BB T-cell activation molecule eradicate established tumors. Nat Med. 1997;3(6):682-685.

13. Sznol M, et al. Phase I study of BMS-663513, a fully human anti-CD137 agonist monoclonal antibody, in patients with advanced cancer. J Clin Oncol. 2008;26(15S):3007.

14. Dubrot J, et al. Treatment with anti-CD137 mAbs causes intense accumulations of liver $\mathrm{T}$ cells without selective antitumor immunotherapeutic effects in this organ. Cancer Immunol Immunother. 2010;59(8):1223-1233

15. Seo Y, et al. Cetuximab-mediated ADCC activity is correlated with the cell surface expression level of EGFR but not with the KRAS/BRAF mutational status in colorectal cancer. Oncol Rep. 2014;31(5):2115-2122.

16. Quesnelle KM, Wheeler SE, Ratay MK, Grandis JR. Preclinical modeling of EGFR inhibitor resistance in head and neck cancer. Cancer Biol Ther. 2012;13(10):935-945.

17. Yamashita M, Chattopadhyay S, Fensterl V, Saikia P, Wetzel JL, Sen GC. Epidermal growth factor receptor is essential for Toll-like receptor 3 signaling. Sci Signal. 2012;5(233):ra50.

18. Kumai $T$, et al. EGFR inhibitors augment antitumour helper T-cell responses of HER family-specific immunotherapy. BrJ Cancer. 2013;109(8):2155-2166.

19. Shay $T$, et al. Conservation and divergence in the transcriptional programs of the human and mouse immune systems. Proc Natl Acad Sci U S A. 2013;110(8):2946-2951.

20. Shultz LD, Brehm MA, Garcia-Martinez JV, Greiner DL. Humanized mice for immune system investigation: progress, promise and challenges. Nat Rev Immunol. 2012;12(11):786-798.

\title{
Sex, drugs, and trial design: sex influences the heart and drug responses
}

\author{
Elizabeth Murphy ${ }^{1}$ and Charles Steenbergen ${ }^{2}$ \\ ${ }^{1}$ National Heart, Lung, and Blood Institute, NIH, Bethesda, Maryland, USA. \\ 2Department of Pathology, The Johns Hopkins Medical Institutions, Baltimore, Maryland, USA.
}

\begin{abstract}
Preclinical studies indicate that the phosphodiesterase 5 (PDE5) inhibitor sildenafil is protective against hypertrophy-induced cardiac remodeling. Despite an initial clinical study demonstrating sildenafil-dependent amelioration of pathological remodeling, the cardioprotective effect of this drug was not significant in a large placebo-controlled clinical trail. In this issue, Sasaki and colleagues reveal that the efficacy of PDE5 inhibition in female mice requires estrogen. Induction of cardiac stress in male and intact female mice resulted in increased activation of protein kinase G (PKG) signaling, which was further enhanced by sildenafil. PKG activity was not enhanced in ovariectomized (OVX) female mice as a result of cardiac stress, but administration of estrogen restored PKG activation and enhancement by sildenafil. These data highlight the importance of considering sex-specific differences and drug responses in clinical trial design.
\end{abstract}

\section{Regulation of cGMP levels in the heart}

An increase in nitric oxide (NO) or activation of natriuretic peptide triggers guanylyl cyclase (sGC), resulting in the generation of cGMP, which in turn promotes protein kinase $\mathrm{G}(\mathrm{PKG})$ signaling to regulate multiple cellular processes, including the cardiac response to stress. In addition to sGC, phosphodiesterases (PDEs) regulate cGMP levels via degradation (see Figure 1). While many PDEs are promiscuous and can degrade either cAMP or cGMP (1), PDE5 is reported to specifically degrade cGMP and is inhibited by the drug sildenafil. Under basal conditions, levels of PDE 5 are low in the heart; therefore, PDE5 inhibition with sildenafil has

Conflict of interest: The authors have declared that no conflict of interest exists.

Citation for this article: J Clin Invest. 2014; 124(6):2375-2377. doi:10.1172/JCI76262. little effect on cGMP and PKG activation (2). In contrast, conditions that induce or are associated with hypertrophic stress, including overexpression of the $G$ protein $\mathrm{Gq}$, transaortic constriction, or natriuretic peptide signaling, lead to increased sGC activity, subsequent cGMP production, and activation of PKG, which phosphorylates and activates a number of targets. PKG signaling is thought to reduce hypertrophic remodeling, and previous studies (3-5) have shown that PDE5 inhibition by drugs such as sildenafil can ameliorate the pathological cardiac remodeling that is associated with hypertrophy and heart failure (4-6). Conversely, overexpression of PDE5 in mice promotes pathological remodeling in the heart in the absence of cardiac stress $(3,7)$. Compared with tissue from healthy subjects, PDE5 expression is upregulated in patients with right ventricular failure (8). Furthermore, a small clinical trial demonstrated that silden- afil reduces pathological remodeling in patients with systolic heart failure (9); importantly, however, a large multicenter, placebo-controlled trial (RELAX) did not show a beneficial effect of sildenafil in the treatment of heart failure with preserved ejection fraction (10).

\section{Sex matters: sex-dependent responses to cardiac stress}

Although sex-associated differences are increasingly recognized in nonreproductive physiology, metabolism, and disease, these differences are generally not taken into account during clinical trial design or in drug treatment (11); however, this paradigm is beginning to change. For example, just last year the FDA recommended that women receive a different (lower) dose of zolpidem, a drug used to treat insomnia, than that recommended for men (12). In the FDA drug safety announcement about the new zolpidem dosing recommendations for women, it was noted that "women appear to be more susceptible to this risk (next morning impairment) because they eliminate zolpidem from their bodies more slowly than men." This example highlights that sex-dependent drug effects need to be taken into consideration when developing dosage guidelines.

There have been numerous sex-specific differences reported in cardiovascular physiology and disease (13). The majority of preclinical studies showing a beneficial effect of PDE5 inhibition on adverse remodeling were performed on male ani- 